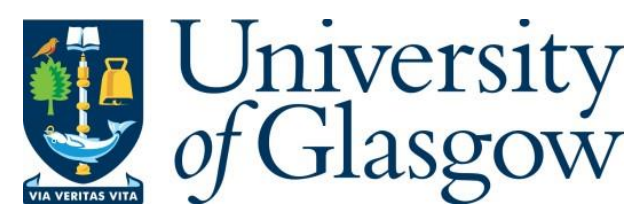

Firstbrook, D., Worrall, K., Timoney, R. and Harkness, P. (2018) Ultrasonically

Assisted Hammer-Action Penetrators in Planetary Regolith. ASCE Earth and Space Conference, Cleveland, OH, USA, 10-13 Apr 2018.

There may be differences between this version and the published version. You are advised to consult the publisher's version if you wish to cite from it.

http://eprints.gla.ac.uk/156625/

Deposited on: 2 February 2018

Enlighten - Research publications by members of the University of Glasgow http://eprints.gla.ac.uk 


\title{
Ultrasonically Assisted Hammer-Action Penetrators in Planetary Regolith
}

\author{
David Firstbrook, ${ }^{1}$ Kevin Worrall, ${ }^{1}$ Ryan Timoney, ${ }^{1 *}$ and Patrick Harkness ${ }^{1}$. \\ ${ }^{1}$ University of Glasgow, University Avenue, Glasgow, Scotland, United Kingdom; \\ PH (0044) 141330 3233; \\ email: patrick.harkness@glasgow.ac.uk. \\ *indicates student author.
}

\section{ABSTRACT}

This paper describes the effects of combining ultrasonic vibration with hammeraction style penetrators, such as the $\mathrm{HP}^{3}$ probe on the InSight mission. By synchronizing short pulses of vibration with the impact of the hammer, the number of hammer strikes required to reach a specified depth was reduced by over a third in some cases, depending on the sand and amplitude of vibration used. Additional investigations looked at comparing the performance of pure hammering with pure pulsing, allowing recommendations for operational procedures if this technology were to be taken forward in the future.

\section{INTRODUCTION}

Access to the subsurface of planetary bodies is an area of key scientific interest. On Mars, the surface erosion rate is low enough that even at relatively shallow depths of a few meters, it is possible to find pristine samples of soil and rocks that have been untouched for millions of years, allowing us an unrivalled glimpse at the historical conditions on the planet.

Self-propelling penetrating moles are an attractive option for accessing these depths, offering tens of meters depth at a fraction of the required overhead weight. This is particularly valuable for small planetary bodies, due to the low surface gravity and thus lower available weight. These moles utilize an internal mass to hammer the probe through the granular surface, and have been used in several missions such as Beagle-2, the Philae lander, and the 2018 InSight mission (Glaser, Ball, and Zacny 2008; Hansen-Goos and Grott 2014). A balance between progression rate and structural damage to the internal components of the probe is essential, as impulses can reach potentially destructive levels, as demonstrated with the PLUTO probe aboard Beagle-2 exhibiting hammer strikes as high as $8,000 \mathrm{~m} / \mathrm{s}^{2}$ (Pinna et al. 2001; Glaser, Ball, and Zacny 2008).

Parallel to this, ultrasonic vibration has been shown to reduce overhead force requirements under progression through granular material (Firstbrook et al. 2017). The tests concentrated on static penetration of a titanium penetrator, tuned to vibrate resonantly at $20 \mathrm{kHz}$. The probe was excited with vibration amplitudes between $0-5$ $\mu \mathrm{m}$, with higher amplitudes displaying greater reductions in peak penetration forces, albeit with diminishing returns. In some sands, the forces were reduced by up to an 
order of magnitude. The principle in this lies in the fact that the ultrasonic vibration causes a very localized region of fluidization in the granular material immediately surrounding the probe, reducing surface friction and therefore penetration forces.

It is believed that by utilizing the force reduction properties of ultrasonic vibration, a new hybrid mole hammering technique could be created that reduces both the internal impact forces, as well as enabling larger penetration depths. The work described in this paper lays out the first steps taken to investigate this experimentally, including an analysis of the results and a discussion of the implications.

\section{EXPERIMENTAL DESIGN}

Parameters. Hammering probes for use in low-gravity environments utilize springs to deliver the required impact, requiring careful considerations of the reacted forces as these can cause the penetrator to jump out of the hole (Glaser, Ball, and Zacny 2008). These probes incorporate suppressors into the spring systems to reduce the reacted force below the skin friction, however this would require significant development and optimization to be used for these tests. Instead, a simple sliding weight was used for these tests, using the gravity of Earth to provide the acceleration and impact forces. The development of the $\mathrm{HP}^{3}$ mole determined that a total hammer impact energy of $0.8 \mathrm{~J}$ was required to fulfil the depth requirements of the mission (Hansen-Goos and Grott 2014). To replicate this energy, the $952.4 \mathrm{~g}$ hammer would need to fall a distance of $8.6 \mathrm{~cm}$.

Two regolith simulants were chosen for these experiments due to the high degree of characterization and heritage with ultrasonic tests. These were BP, a block-paving sand used for control from the University of Glasgow; and SSC-3, a beach sand used by the Surrey Space Centre in test beds for the development of planetary rovers (Yeomans and Saaj 2014). Both sands are quartz based with a uniform distribution, and prepared to a medium-high relative density. A summary of the properties and densities of the sands used for these tests, as well as the standard deviation of the density values, are given in Table 1 . For a full characterization and description of their interactions with ultrasonic probes, please refer to Firstbrook et al. 2017 and Firstbrook 2017. Each container of sand was prepared using a vibrating container and filled from a height of $1 \mathrm{~m}$, producing a consistent sample each time that was verified with several static penetration tests.

Table 1. Overview of the two regoliths used.

\begin{tabular}{ccccccc}
\hline Sand & $\begin{array}{c}\text { Bulk density } \\
(\mathrm{g} / \mathrm{cc})\end{array}$ & $\begin{array}{c}\text { Standard } \\
\text { deviation }\end{array}$ & $\begin{array}{c}\text { Relative } \\
\text { density }(\%)\end{array}$ & $\begin{array}{c}\text { Friction } \\
\text { angle }\left(^{\circ}\right)\end{array}$ & Size & Shape \\
\hline BP & 1.631 & 0.000615 & 48.3 & 30.3 & Medium & $\begin{array}{c}\text { Sub- } \\
\text { rounded } \\
\text { Sub- } \\
\text { SSC-3 }\end{array}$ \\
\hline
\end{tabular}


A container size of $14 \mathrm{~cm} \times 14 \mathrm{~cm} \times 25 \mathrm{~cm}$ was chosen to allow a sufficiently large container/probe diameter ratio (D/d of 8.1 on the edge and 11.5 on the diagonal) to avoid wall effects (Seguin, Bertho, and Gondret 2008), whilst also providing a volume of sand small enough so that the vibrating motor would be able to fully fluidize all regions within the container during/after filling. To fully examine the effects of ultrasonic vibration, a range of excitation amplitudes from $0.4-1.6 \mu \mathrm{m}$ were used, with any non-ultrasonic test designated with an amplitude of $0 \mu \mathrm{m}$. The vibration was pulsed for a short duration, and needed to be activated at the same time as the release of the hammer. As the hammer would theoretically take $132 \mathrm{~ms}$ to fall $8.6 \mathrm{~cm}$, a pulse duration of $400 \mathrm{~ms}$ was eventually decided upon, allowing sufficient time for the hammer to drop, as well as for the penetrator to travel after being struck by the hammer. The depth of the penetrator after each impact was measured by eye with a needle point and a fixed ruler, giving an accuracy to the nearest $0.5 \mathrm{~mm}$.

Equipment. The ultrasonic vibration was provided by a Sonic Systems ${ }^{\mathrm{TM}}$ L500 transducer, powered by a P100 ultrasonic power supply which could track and provide an excitation signal between $19.5 \mathrm{kHz}-20.5 \mathrm{kHz}$. The penetrator was a titanium probe tuned to vibrate at $20 \mathrm{kHz}$, with a $201 \mathrm{~mm}$ length, $17.25 \mathrm{~mm}$ diameter shaft, and $60^{\circ}$ cone tip, shown in Figure 1. The shape of the probe, or horn, amplified any excitation signal by a factor of 3.5 , known as the 'gain' of the horn.

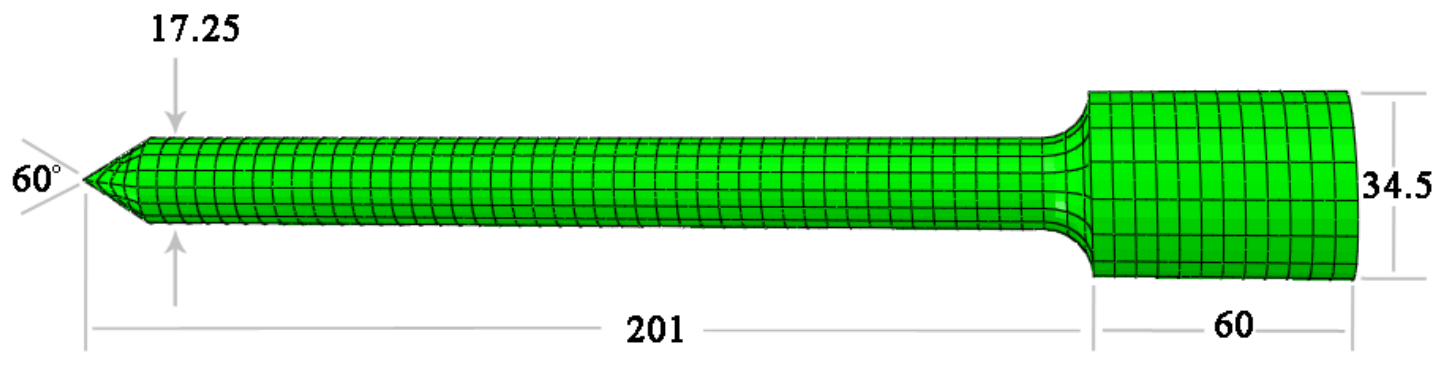

Figure 1. Size and shape of the ultrasonic penetrator used. Dimensions are given in $\mathbf{m m}$.

Both the hammer and the anvil were manufactured from mild steel, and a hole through the center of the hammer allowed it to slide vertically along a loose-fitting rod, ensuring that the striking contact was always flush. An electromagnet, fixed to the top of an aluminum support, provided the catch-and-release mechanism for the hammer, ensuring it could be released electronically with accurate timing and consistent starting position, enabling consistent impact energies of $0.8 \mathrm{~J}$. The hammer was returned by hand to the electromagnet between each hammer blow.

The penetrator and hammer rig was free to move vertically along a rail using a linear bearing attached to one side of the anvil. The friction between the bearing and the vertical rail was measured to be $0.4 \mathrm{~N}$, and was considered negligible and left out of further analysis. The whole experimental apparatus can be seen in Figure 2. 


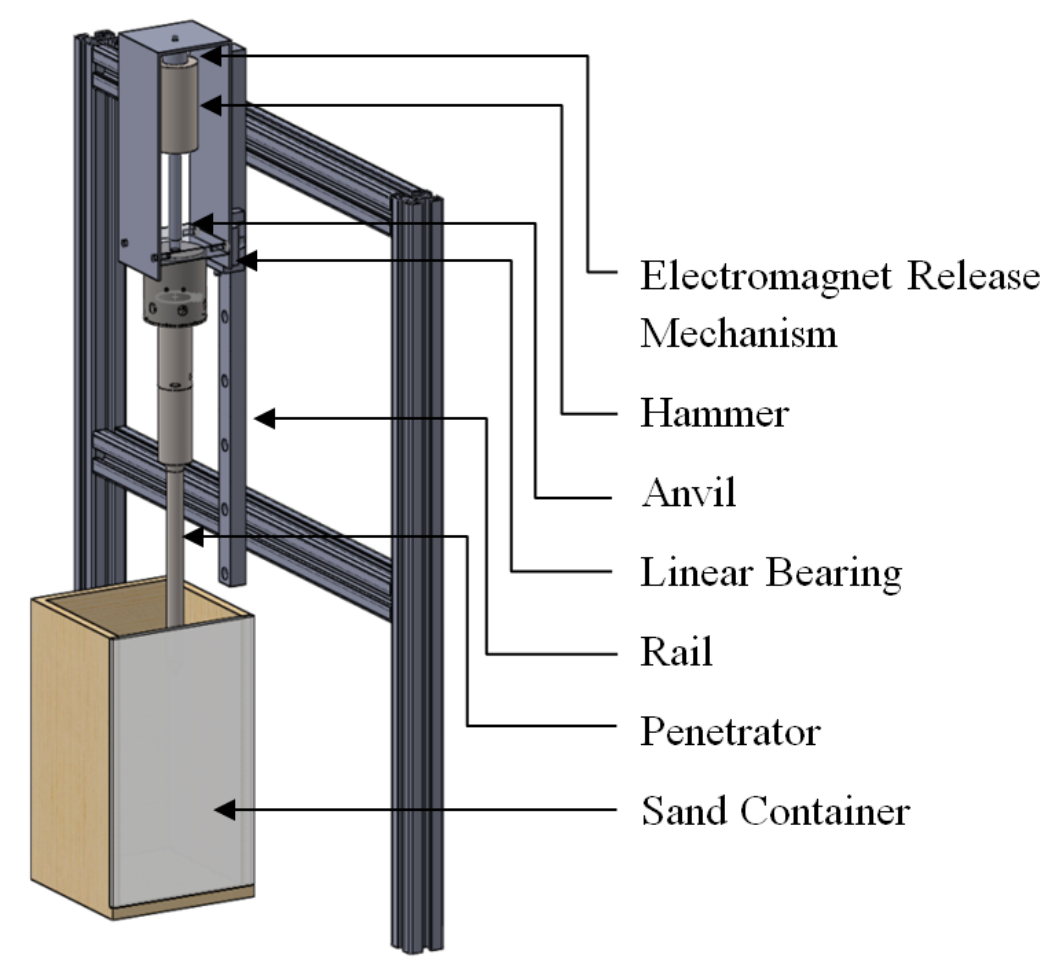

Figure 2. Overview of the hammer rig. Most of the supporting aluminum frame has been removed for ease of viewing.

Procedure. Two main experiments are described in this paper; Firstly, where ultrasonic pulsing and hammering occur simultaneously, and secondly, where purely ultrasonic pulsing was run in order to compare the differences. For each experiment, a fresh sample of sand was prepared in the same manner, so that each sample was as consistent as possible between runs. Stoppers on the table guaranteed that the container was positioned in the same place each time, ensuring the penetrator consistently travelled through the center of the container.

The penetrator was then gently lowered into the sand until it came to its resting, roughly $41 \mathrm{~mm}$ for BP and $51 \mathrm{~mm}$ for SSC-3. These form the initial starting points for each experiment. The value of ultrasonic vibration was set, and the hammer raised to the active electromagnet until it was held in position. Using a custom Arduino code for data acquisition, the ultrasonic power was measured for $200 \mathrm{~ms}$ (to get a baseline reading in case of any DC offset), then the ultrasonic vibration was initiated and the electromagnet deactivated simultaneously, allowing the hammer to fall. The ultrasonic vibration was turned off after $400 \mathrm{~ms}$ and the electromagnet turned back on. Ultrasonic power was measured for an additional $200 \mathrm{~ms}$ (for off-set purposes), and then the hammer was raised back to the magnet by hand, ready to repeat the process. For brevity, the hammering-only tests are referred to as the " $0 \mu \mathrm{m}$ " runs to represent no ultrasonic vibration. A timeline of this process is provided in Figure 3. The purely ultrasonic pulsing tests follow the same procedure, but the hammer was not raised to the electromagnet and thus hammering did not occur. 


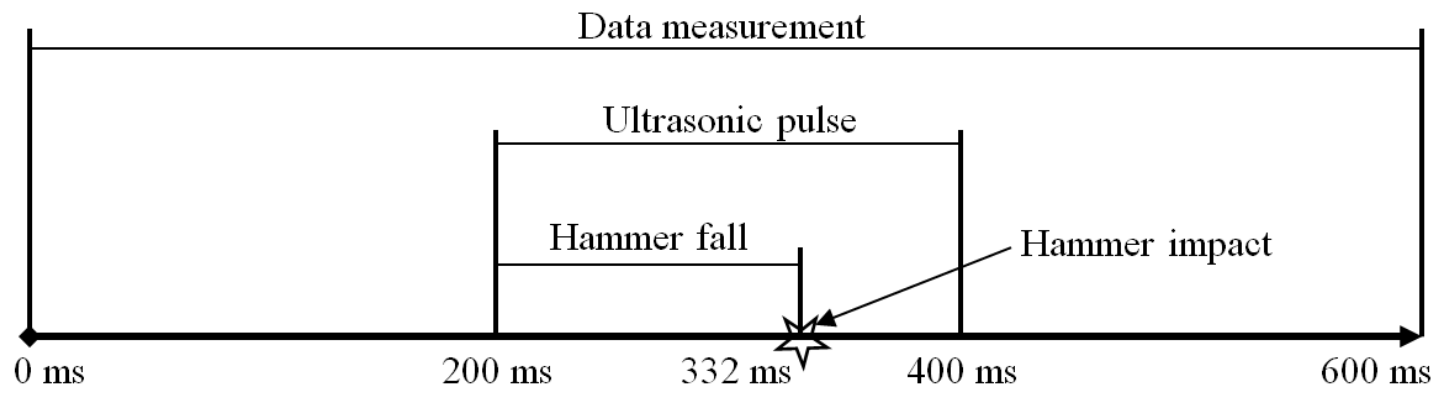

Figure 3. Timeline of the hammering process. The hammer was calculated to fall the $8.6 \mathrm{~cm}$ distance in $132 \mathrm{~ms}$.

\section{RESULTS AND DISCUSSION}

Unfortunately, due to a low sampling rate and a high degree of noise in the system, the power measurement results proved to be insufficient for any scientific analysis, but remained on the order of $1-2 \mathrm{~W}$. A significant amount of data about the penetration depth, however, was gained for both the simultaneous and pulsing experiments, which will be discussed here.

Simultaneous hammering and ultrasonics. The penetration depth as a function of hammer blows in BP and SSC-3 are shown in Figure 4 (a) and (b) respectively. Predictably, the higher values of ultrasonic amplitude resulted in fewer hammer strikes to reach the final depth of $190 \mathrm{~mm}$. Additionally, more strikes were required with BP than SSC-3 for non-ultrasonic hammering, which is in agreement with the higher relative density of BP (48.2\% compared to $43.1 \%)$, as well as the higher peak penetration forces seen in previous work for static penetration (Firstbrook et al. 2017). When ultrasonic vibration is introduced, there appears to be little difference between BP and SSC-3 in the total strikes required to reach maximum depth. This agrees with previous evidence that shows that BP tends to a higher reactivity to ultrasonic vibration than SSC-3 during static penetration.
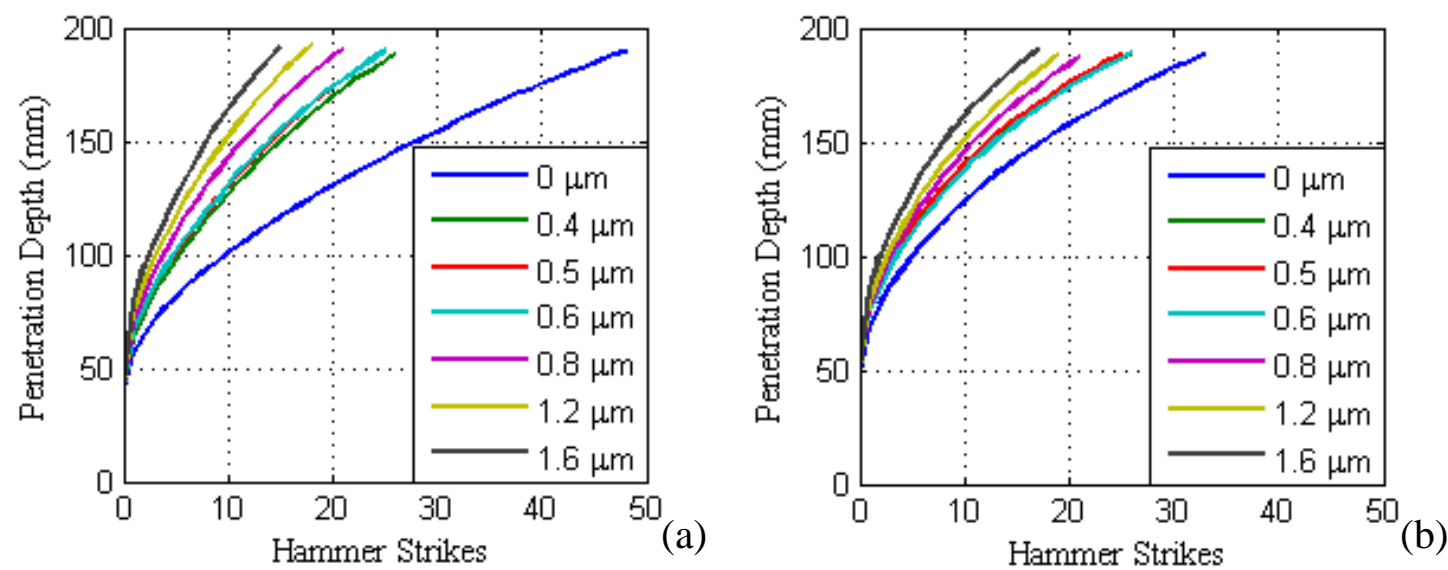

Figure 4. Penetration depth with number of hammer strikes for (a) BP and (b) SSC-3. The amplitude of the synchronized ultrasonic pulse is also given. 
Interestingly, there seems to be very little variance in depth profile for the three lowest amplitudes, 0.4, 0.5, and $0.6 \mu \mathrm{m}$. This feature was not noted until after the experiments were completed and the data plotted and analyzed, and was originally thought to be an issue with the experimental set-up. The apparatus was checked, especially the Arduino code that governed the amplitude command to ensure it was not sending an incorrect amplitude signal, but no issue was found. Additionally, not only was this effect seen in both SSC-3 and BP, but each run was also repeated three times and averaged, and each repetition showed the same result.

The general trend is that an increasing ultrasonic amplitude results in a larger depth of penetration at the same number of hammer strikes, however it is clear that an inflection point appears in the range of $0.4-0.6 \mu \mathrm{m}$. Here, the depth per strike levelsoff to some degree, and in some cases actually can be seen to decrease slightly with increasing amplitude, such as the shallower curve of the $0.6 \mu \mathrm{m}$ run in Figure 4 (b) compared to the $0.5 \mu \mathrm{m}$ run. If it had occurred in just one of the runs then it could be put down to slight differences in the final bulk density of the sample of sand. However, with this effect being present across all runs, it is more likely that this is a genuine effect of the amplitude of ultrasonic vibration, and would require further investigation should this technology be taken forward.

An interesting effect is that the depth profiles of the ultrasonic cases appear to be very similar between BP and SSC-3, especially in the range $0.4-0.8 \mu \mathrm{m}$. Since the purely-hammering runs are quite different (48 impacts required in BP compared to 33 impacts in SSC-3 to reach 190mm depth), it is likely that this is a coincidence rather than an intrinsic property of ultrasonics and granular material. However, further tests into a broader range of soils should be conducted in the future to test this.

A method of comparing the hammering penetration performance, as used in the development of the InSight probe HP3 (Hansen-Goos and Grott 2014), is to calculate the advancement per stroke of the probe, known as the dynamic cone penetration index (DCPI, or occasionally DPI). This is shown for BP and SSC-3 in Figure 5 (a) and (b) respectively.

Taking BP as an example, the probe at its deepest point $(190 \mathrm{~mm})$, the rate of advancement was $5 \mathrm{~mm}$ per strike when using a vibration amplitude of $1.6 \mu \mathrm{m}$, and $1.83 \mathrm{~mm}$ per strike when using $0 \mu \mathrm{m}$ amplitude (the non-ultrasonic case). This leads to an increase in penetration performance by a factor of $\sim 2.7$ when using ultrasonic vibration at this depth. The equivalent value for SSC-3 is a factor of $\sim 1.6$, so it is clear that the specific substrate in question plays a large role in the effectiveness of ultrasonic vibration. 


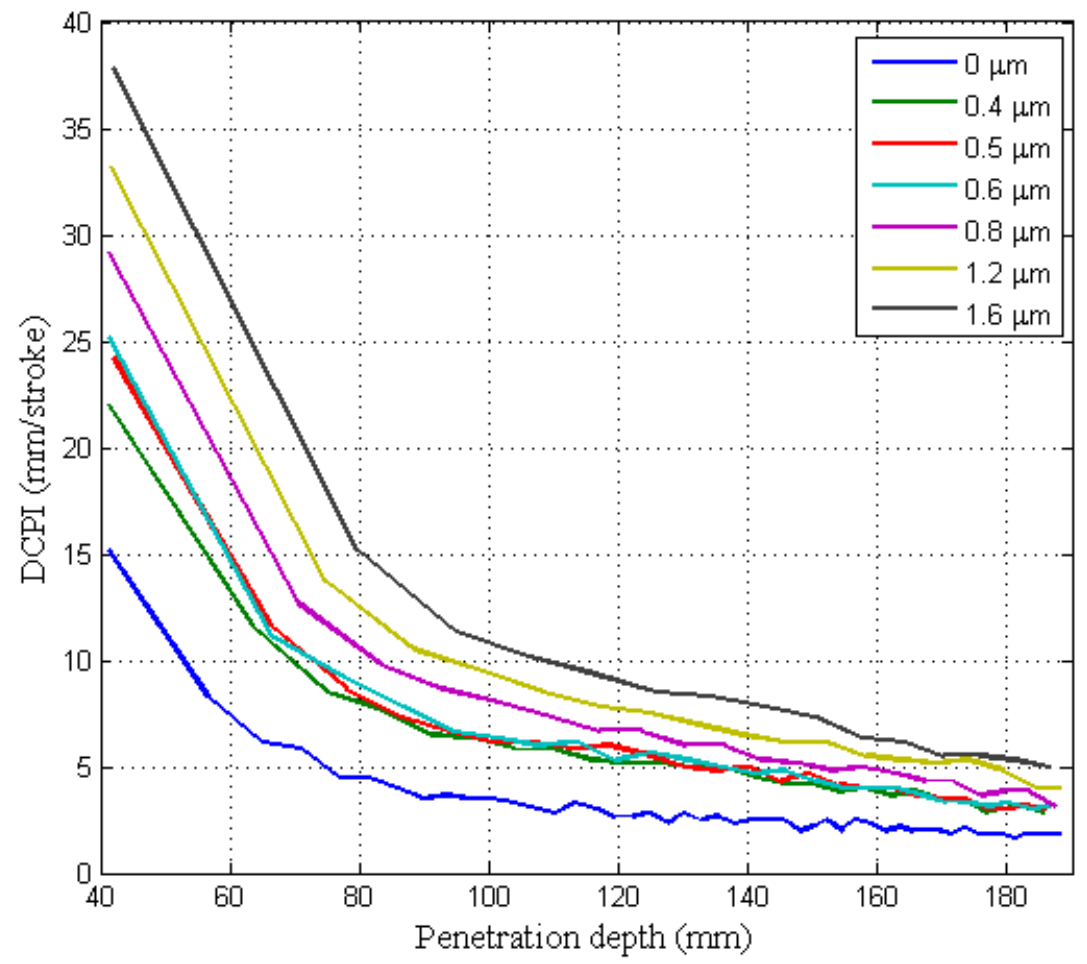

(a)

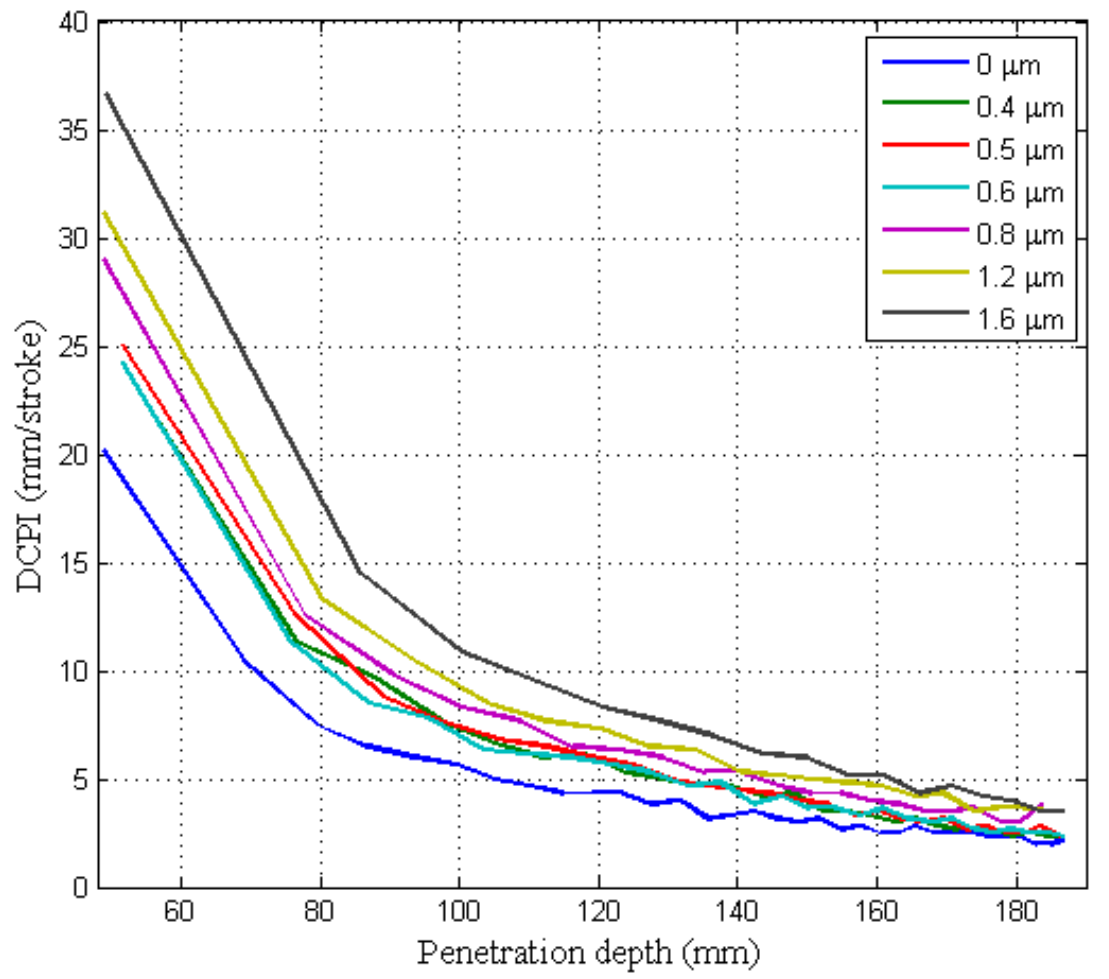

(b)

Figure 5. DCPI as a function of depth at various ultrasonic excitation amplitudes for (a) BP and (b) SSC-3. 
Ultrasonic pulsing. To discern the specific contribution that ultrasonic vibration has, equivalent tests were conducted that used the same duration of ultrasonic pulsing but without any hammering impact. The penetration of purely ultrasonic pulsing was much less efficient, and over the span of 50 pulses the maximum achievable depth was just $150 \mathrm{~mm}$, occurring in $\mathrm{BP}$ at $1.6 \mu \mathrm{m}$.

It is clear that the combination of hammering and ultrasonic vibration results in a more efficient penetration than either of the techniques on their own. However, it is useful to determine how the two methods interact with each other, and whether the combination of hammering and ultrasonics is greater than the sum of them separately. To visualize this, the following graphs each display results of each of the six different ultrasonic amplitudes, shown in Figure 6 for BP and Figure 7 for SSC-3. Each graph contains four plots, showing the penetration depth profile of pure hammering, pure ultrasonic pulses, both methods together (referred to as the 'simultaneous' runs), and finally the sum of the penetration profiles of both (referred to as the 'summed' runs). These 'summed' runs are a theoretical concept to aid with comparisons, and are calculated by summing the increase in depth after the penetrator has settled under its own weight; between $41-42 \mathrm{~mm}$ for BP, and $49-51 \mathrm{~mm}$ depth for SSC-3.

For BP, the difference between the simultaneous runs and the summed runs is remarkably small, reaching the final penetration depth with little difference in the number of strikes and/or pulses. The gradient between the two varies slightly, with the summed results showing a slightly faster penetration initially. SSC-3 on the other hand displays a significant difference in every case, with the summed results reaching maximum penetration depth between 5-8 strikes/pulses earlier than the simultaneous results. The shape of the curve, however, is much more similar between the two methods, with the summed results at $0.4 \mu \mathrm{m}$ excitation amplitude matching very well with the simultaneous results at $0.8 \mu \mathrm{m}$. These results indicate that whilst simultaneous hammering and ultrasonic does reduce the number of strikes needed to reach a given depth, there is some loss of penetration efficiency when they are combined together. A small loss in efficiency, however, would not discount this as a viable option, as the most important factor is that the total number of strikes is reduced, thereby reducing stresses and damage potential in a final tool.

Whilst the final penetration depth of pure ultrasonic pulsing is far below that of pure hammering, it is interesting to investigate differences at shallow depths. What can be seen in some figures, particularly for the $0.8-1.6 \mu \mathrm{m} \mathrm{BP}$ and the $1.6 \mu \mathrm{m} \mathrm{SSC}-3$ runs, is that initially the purely pulsing technique can out-perform the purely hammering technique at a low number of strikes/pulses, but at larger number of pulses the depth gained per pulse is too small to match the capabilities of the hammering technique. This is seen starting at lower amplitudes in BP, which along with the greater overall penetration depth over SSC-3 could suggest that BP reacts more favorably to ultrasonic penetration than SSC-3 does. The opposite appears to be true for nonultrasonic hammering, as the full depth was reached in 50 strikes for BP, whilst only requiring 33 strikes for SSC-3. The specific reason for this is unknown, and could be due to a variety of reason such as bulk density, relative density, particle sizes etc. 

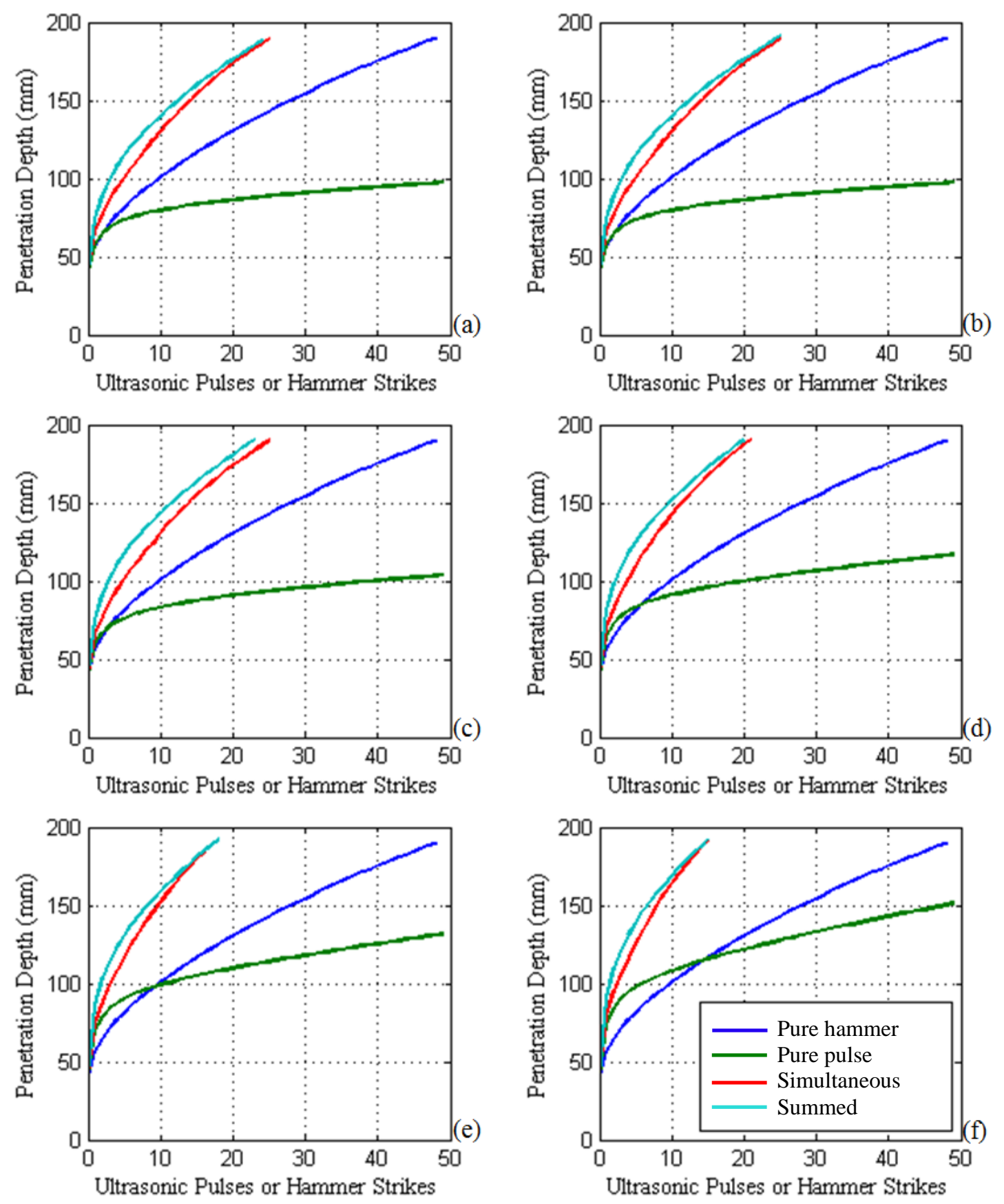

Figure 6. Simultaneous hammer strike and ultrasonic pulsing, pure striking pure pulsing, and the sum of the two in BP using an excitation amplitude of (a) 0.4 $\mu \mathrm{m}$, (b) $0.5 \mu \mathrm{m}$, (c) $0.6 \mu \mathrm{m}$, (d) $0.8 \mu \mathrm{m}$, (e) $1.2 \mu \mathrm{m}$, and (f) $1.6 \mu \mathrm{m}$. 

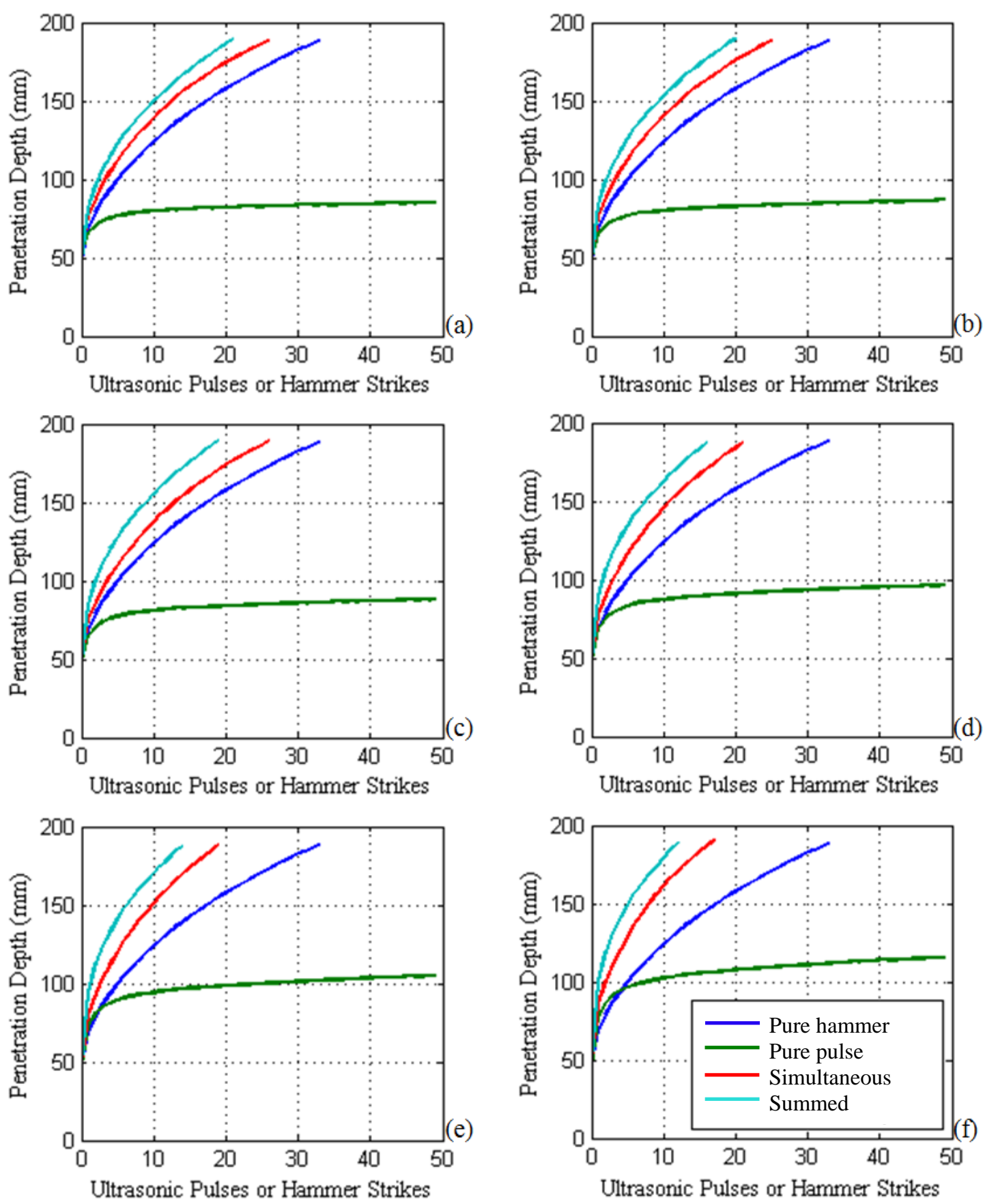

Figure 7. Simultaneous hammer strike and ultrasonic pulsing, pure striking pure pulsing, and the sum of the two in SSC-3 using an excitation amplitude of (a) 0.4 $\mu \mathrm{m}$, (b) $0.5 \mu \mathrm{m}$, (c) $0.6 \mu \mathrm{m}$, (d) $0.8 \mu \mathrm{m}$, (e) $1.2 \mu \mathrm{m}$, and (f) $1.6 \mu \mathrm{m}$. 


\section{CONCLUSION}

This paper has built upon previous investigations into the phenomena associated with ultrasonic vibration and granular material, and applied it to penetration techniques that are currently used in space missions. As such, these experiments take the first steps into using direct ultrasonic vibration as a viable device for penetration granular material.

The combination of simultaneous hammering and ultrasonic pulsing showed that the addition of ultrasonic vibration can reduce the total number of strikes required on a probe to reach a specific depth, in this case $190 \mathrm{~mm}$. In BP this was reduced from 48 strikes to 15 strikes at the highest excitation amplitude of $1.6 \mu \mathrm{m}$, and from 33 strikes to 17 strikes in SSC-3. Reducing the number of strikes for a hammering device can significantly extended its operational lifetime, and this technique could potentially be used to great effect in this regard. From the view point of penetration rate per strike as a function of depth (DCPI), $1.6 \mu \mathrm{m}$ of vibration increased penetration performance by a factor of $\sim 2.7$ in $\mathrm{BP}$, and 1.6 in SSC-3.

Additionally, it was discovered that at low penetration depths, ultrasonic vibration can fluidize the sand to such an extent that the penetrator undergoes a brief moment of near free-fall, where it progresses for a short time unhindered. This effect was not seen at larger depths, where progression due to ultrasonic vibration was much more linear. In applications for a probe that is allowed to progress through terrain under its own weight, it would be beneficial to extend the duration of ultrasonic vibration at shallower depths to take advantage of this effect. This will no doubt be heavily affected by the local gravity, and further tests could be done to investigate the relationship between gravity and ultrasonic pulse duration.

\section{REFERENCES}

Firstbrook, David. 2017. "Ultrasonically Assisted Penetration Through Granular Materials for Planetary Exploration." University of Glasgow. http://theses.gla.ac.uk/id/eprint/8256.

Firstbrook, David, Kevin Worrall, Ryan Timoney, Francesc Suñol, Yang Gao, and Patrick Harkness. 2017. "An Experimental Study of Ultrasonic Vibration and the Penetration of Granular Material." Proceedings of the Royal Society A 473 (2198): 1-16. doi:10.1098/rspa.2016.0673.

Glaser, David L, Andrew J Ball, and Kris A Zacny. 2008. "A Review of Penetrometers for Subsurface Access on Comets and Asteroids." Meteoritics \& Planetary Science 43 (6): 1021-32. doi:10.1111/j.1945-5100.2008.tb00690.x.

Hansen-Goos, H, and M Grott. 2014. "Predicted Penetration Performance of the InSight HP3 Mole.” In 45th Lunar and Planetary Science Conference, 1325. Houston, Texas. http://adsabs.harvard.edu/abs/2014LPI....45.1325H.

Pinna, S., F. Angrilli, H. Kochan, and L. Richter. 2001. "Development of the Mobile Penetrometer (Mole) as Sampling Tool for the Beagle2 Lander on Mars Express 2003." Advances in Space Research 28 (8): 1231-36. doi:10.1016/S0273- 
1177(01)00300-3.

Seguin, Antoine, Yann Bertho, and Philippe Gondret. 2008. "Influence of Confinement on Granular Penetration by Impact." Physical Review E Statistical, Nonlinear, and Soft Matter Physics 78 (1): 1-4. doi:10.1103/PhysRevE.78.010301.

Yeomans, Brian, and Chakravathini M Saaj. 2014. "Towards Terrain Interaction Prediction for Bioinspired Planetary Exploration Rovers." Bioinspiration \& Biomimetics 9 (1). IOP Publishing: 16009. doi:10.1088/1748-3182/9/1/016009. 